\title{
Bivalent BRD4 Inhibitor AZD5153
}

National Cancer Institute

\section{Source}

National Cancer Institute. Bivalent BRD4 Inhibitor AZD5153. NCI Thesaurus. Code C151943.

An orally bioavailable bivalent inhibitor of bromodomain-containing protein 4 (BRD4), with potential antineoplastic activity. Upon oral administration, the BRD4 inhibitor AZD5153 selectively binds to the acetylated lysine recognition motifs in two bromodomains in the BRD4 protein, thereby preventing the binding of BRD4 to acetylated lysines on histones. This disrupts chromatin remodeling and dysregulates expression of target genes, which leads to the downregulation of the expression of certain growth-promoting genes, induces apoptosis and inhibits the proliferation of BRD4-overexpressing tumor cells. BRD4, a member of the human bromodomain and extra-terminal (BET) family of proteins, is a transcriptional regulator that is overexpressed in certain tumor cells and plays an important role in cellular proliferation. 